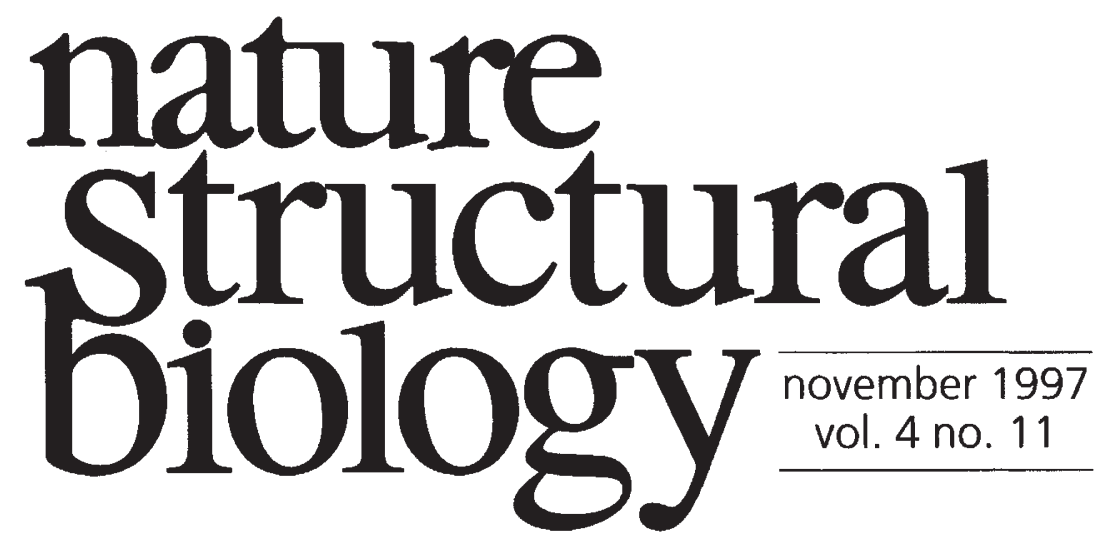

\title{
Connecting up the cytoskeleton
}

For such a small protein, profilin $\left(12,000-15,000 M_{\mathrm{r}}\right)$ has turned out to highly enigmatic ${ }^{1}$. Although it was one of the first actin-monomer binding proteins to be characterized, its role in remodelling the cytoskeleton - and in particular its role as a link between the various signal transduction pathways and the cytoskeleton - has only slowly come into focus over the last several years. Steven Almo and colleagues now provide a further sharpening of the image of the protein in this issue of Nature Structural Biology ${ }^{2}$, where they report the structure of profilin bound to poly-L-proline, a complex that is expected to mimic profilin's interaction with a rapidly growing list of proline-rich cytoskeletal partners.

Cells are highly structured, containing a resilient endoskeleton composed of microtubules, intermediate filaments and actin filaments. Yet, in terms of their mobility and morphology, cells are often highly dynamic, dramatically rearranging the components of their cytoskeletons in minutes in response to external stimuli. The mechanical forces underlying these dramatic structural changes are derived in large part from the actin-filament based component of the cytoskeleton.

Profilin's role in the dynamics of actin polymerization and depolymerization is complex and, as yet, not fully understood. Actin is in equilibrium between monomers (G-actin) and filaments (F-actin) and has a critical concentration (the concentration above which it spontaneously forms filaments) ranging from $\sim 0.2$ to $\sim 2.0 \mu \mathrm{M}$. Yet the concentration of actin monomers in many cells ranges from $50-200 \mu \mathrm{M}$. How is this apparent disequilibrium maintained? In vitro profilin is able to sequester actin monomers, preventing filament formation, initially suggesting that this may be profilin's function in the cell. In fact, profilin only plays a minor role (if at all) in sequestering actin; instead, the even smaller, $\sim 5,000 M_{\mathrm{r}}$ actin binding protein, thymosin $\beta 4$, is responsible in large part for the packaging of monomeric actin in the cell ${ }^{3}$.

Actin is an ATPase; binding and hydrolysis of ATP is an essential part of the mechanism of filament formation ${ }^{4}$. Profilin promotes the release of adenine nucleotide from actin, decreasing actin's affinity for nucleotide by up to 1,000-fold. Since ATP-actin has a critical concentration that is an order of magnitude less $(\sim 0.2 \mu \mathrm{M})$ than that of ADP-actin $(\sim 2.0$ $\mu \mathrm{M})$, profilin's nucleotide exchange has the theoretical potential to promote F-actin formation ${ }^{4}$. However, given that thymosin $\beta 4$ has a 50 -fold higher affinity for ATP-actin over ADP-actin, much of the pool of sequestered actin monomers may already be bound to ATP, and, therefore, this may render profilin's nucleotide exchange activity of little importance in the cells.

A potentially more efficacious profilin activity (in terms of its influence on filament formation) is it's direct effect on the critical concentration of actin ${ }^{6}$. In the presence of profilin it is easier to add ATP-actin to the barbed end of F-actin than in its absence, an activity that is apparently independent of its nucleotide exchange function. The two activities combined, though, give profilin the potential to be an effective desequestering agent, promoting local, rapid filament growth ${ }^{6,7}$. The structural basis of the two effects - nucleotide 


\section{IMAGE \\ UNAVAILABLE FOR COPYRIGHT REASONS}

exchange and lowering the critical concentration of ATP-actin - is presently unknown.

Actin isn't the only protein that profilin interacts with. This was suggested some time ago by experiments showing that profilin can bind runs of 8-10 proline residues in peptides ${ }^{1}$. The first in vivo proline-rich protein ligand to be identified was the vasodilator-stimulated phosphoprotein (VASP) ${ }^{8}$. VASP is found along filamentous structures, in the peripheral lamellae of migrating cells, at the site of focal adhesions and concentrated in highly dynamic regions of the cell cortex, a distribution that has similarities to that of profilin. Furthermore, it has a proline rich central domain that is suggested to be responsible for its interaction with profilin. Murine enabled (Mena), another member of the VASP family of proteins, has also been shown to interact with both profilin and regions of the cytoskeleton that are in flux'.

VASP and Mena may provide links to other regulatory proteins as they themselves are capable of binding to proline rich sequences ${ }^{8,9}$. It is through such proline-binding domains that VASP and Mena are thought to bind to ActA, a surface protein of Listeria monocytogenes ${ }^{9}$. The propulsion of this intracellular bacterial pathogen depends on the rapid polymerization of actin filaments at one pole of the bacterium, forming characteristic 'comet tails'. ActA is the sole bacterial protein required to direct the assembly of the comet tails from host-cell actin and actin-binding complexes. VASP and Mena are potentially capable of binding ActA, and other cytoskeleton-associated proteins such as zyxin and vinculin (which also contain ActA-like proline rich motifs), and profilin simultadomains that are known to bind proline-rich motifs: the SH3 domain the WW domain, profilin ${ }^{2}$ and the EVH1 domain from the VASP/Mena family of proteins ${ }^{9}$ The structures of three of these classes are known, and examples are illustrated here: the SH3 domain from C-sre bound to the ligand VSL12 ${ }^{13}$, shown on the top left; the WW domain from the human Yes kinase associated protein 65 (YAP65) bound to GTPPPPYTVG, shown on the bottom left; and profilin ${ }^{2}$ bound to $(P)_{10}$, on the right. Proline-rich peptides are shown in yellow. Protein domains are shown as gray ribbons with aromatic residues involved in binding proline peptides coloured red. Figure kindly provided by Nicole Mahoney².
1. Sohn, R.H. \& Goldschmidt-Clermont, P.J. Bioessays 16, 465-472 (1994).

2. Mahoney, N.M., Janmey, P.A. \& Almo S.C. Nature Stuct. Biol. 4, 953-960 (1997).

3. Safer, D., Golla, R \& Nachmias, V.T. Proc. Natl. Acad. Sci. USA 87, 2536-2540 (1990).

4. Goldschmidt-Clermont, P.J., Mendelsohn, M.E. \& Gibbs, J.B. Curr. Biol. 2, 669-67 (1992)

5. Carlier, M.F., Jean, C., Rieger, K.J., Lenfant, M. \& Pantaloni, D. Proc. Natl. Acad. Sci. USA 90, 5034-5038 (1993).

6. Pantaloni, D. \& Carlier, M.F. Cell 75, 1007-1014 (1993).

7. Theriot, J.A. \& Mitchison, T.J. Cell 75, 835-838 (1993).

8. Reinhard, M. et al. EMBO J. 14, 1583-1589 (1995).

9. Gertler, F.B., Niebuhr, K., Reinhard, M., Wehland, J. \& Soriano, P. Cell 87, 227-239 (1996).

10. Machesky, L.M. \& Hall, A. Trends Cell Biol. 6, 304-310 (1996)

11. Watanabe, N. et al. EMBO J. 16, 3044-3056 (1997).

12. Evangelista, M. et al. Science $276,118-122$ (1997).

13. Yu H. et al. Cell 76, 933-945 (1994).

14. Macias M.J. et al. Nature 382, 646-649 (1996). neously. By this means members of the VASP family could act to recruit profilin to regions of the cell where dynamic actin remodelling is occurring9.

What of the link that profilin forges between the cytoskeleton and signal transduction? For a start, Mena and VASP may provide links to cyclic nucleotide, and c-Abl and c-Src signalling pathways ${ }^{9}$. Furthermore, profilin has been shown to bind four or five molecules of phosphatidylinsitol 4,5-bisphosphate $\left(\mathrm{PIP}_{2}\right)$ providing a connection to $\mathrm{PIP}_{2}$ pathways ${ }^{1}$. Profilin is also coupled to the signal transduction pathways that regulate and remodel the cytoskeleton through the heterotrimeric guanosine triphosphate (GTP) binding protein (G-protein) Rho. Rho family members are involved in the transmission of signalling events that cause the reorganization of the cortical cytoskeleton and control processes such as cell morphogenesis, motility, adhesion and cytokinesis ${ }^{10}$. There is some suggestion that Rho members may stimulate the synthesis of PIP. Thus, since many actin binding proteins are regulated by $\mathrm{PIP}_{2}$ (including profilin), Rho may exert its effect on the cytoskeleton in this manner.

That notwithstanding, it is the formin family of proteins that provide the most direct and persuasive link between Rho and profilin (and, therefore, actin and the cytoskeleton) ${ }^{11,12}$. The formins are involved cell polarization, cytokinesis and vertebrate limb formation (their name being taken from a mouse gene required for the latter). They share two domains of sequence similarity, the first of which contains 5-12 consecutive proline residues. Two members of this family of proteins, yeast Bnilp and mouse $\mathrm{p} 140 \mathrm{mDia}$, have been shown to interact, through distinct sites on their surfaces, with both profilin - most likely through their proline rich segments - and with the activated, GTP-bound form of $\mathrm{Rho}^{11,12}$. Furthermore, Rho, p140mDia and profilin all co-localize in the cytoskeleton ${ }^{11}$.

The structure of profilin bound to poly-L-proline ${ }^{2}$ allows us to infer something of the complexes with the in vivo proline-rich substrates - Bnilp, p140mDia, VASP, and Mena. But beyond this, we need to know how profilin directly modulates the dynamics of actin assembly and how it interacts with PIP $_{2}$; clearly, there is a lot to look forward to. 